\title{
Coupled-cluster theory for three-body Hamiltonians
}

\author{
G. Hagen,${ }^{1,2,3}$ T. Papenbrock,${ }^{1,2}$ D.J. Dean, ${ }^{1}$ A. Schwenk,${ }^{4,5}$ A. Nogga,${ }^{6}$ M. Włoch,${ }^{7}$ and P. Piecuch ${ }^{7}$ \\ ${ }^{1}$ Physics Division, Oak Ridge National Laboratory, P.O. Box 2008, Oak Ridge, TN 37831, USA \\ ${ }^{2}$ Department of Physics and Astronomy, University of Tennessee, Knoxville, TN 37996, USA \\ ${ }^{3}$ Centre of Mathematics for Applications, University of Oslo, N-0316 Oslo, Norway \\ ${ }^{4}$ TRIUMF, 4004 Wesbrook Mall, Vancouver, BC, Canada, V6T $2 A 3$ \\ ${ }^{5}$ Department of Physics, University of Washington, Seattle, WA 98195, USA \\ ${ }^{6}$ Institut für Kernphysik, Forschungszentrum Jülich, D-52425 Jülich, Germany \\ ${ }^{7}$ Department of Chemistry, Michigan State University, East Lansing, MI 48824, USA
}

\begin{abstract}
We derive coupled-cluster equations for three-body Hamiltonians. The equations for the one- and two-body cluster amplitudes are presented in a factorized form that leads to an efficient numerical implementation. We employ low-momentum two- and three-nucleon interactions and calculate the binding energy of ${ }^{4} \mathrm{He}$. The results show that the main contribution of the three-nucleon interaction stems from its density-dependent zero-, one-, and two-body terms that result from the normal ordering of the Hamiltonian in coupled-cluster theory. The residual three-body terms that remain after normal ordering can be neglected.
\end{abstract}

PACS numbers: 21.10.Dr, 21.60.-n, 31.15.Dv, 21.30.-x

\section{INTRODUCTION}

One of the central challenges in nuclear theory is to understand and predict the structure of nucleonic matter based on microscopic nucleon-nucleon (NN) and manynucleon interactions. In recent years, there has been significant progress in exact calculations of ground and excited states of light nuclei based on various high-precision interactions fitted to NN data 1, 2, 3, 4, 5, 6, 7]. These results clearly show that three-nucleon forces (3NFs) contribute significantly: Without 3NFs, the binding energies depend strongly on the NN potential used, which can be traced to scheme and model dependences in any theory restricted to NN interactions. The existence of 3NFs is not surprising, since nucleons are not point particles. There are always virtual excitations (high-momentum nucleons and $\Delta$-isobars) or internal degrees of freedom (quarks and gluons) that have been "integrated out". This directly leads to three- and many-nucleon interactions. The modern understanding is that nuclear interactions are effective interactions and depend on the resolution scale given by a cutoff $\Lambda$ of the effective theory (see for example [8]). Exact calculations are cutoffindependent up to the effects of omitted higher-order interactions, and therefore varying the resolution scale is a powerful tool to analyze the predictive power of theoretical calculations.

The study of 3NFs in systems beyond the lightest nuclei is an important goal. This requires a flexible technique to solve the many-body problem including $\mathrm{NN}$ and $3 \mathrm{~N}$ interactions. Coupled-cluster theory is a promising tool for this endeavor. This method originated in nuclear physics [9, 10] and is today mostly propelled through its importance in quantum chemistry 11, 12]. For reviews, we refer the reader to Refs. [13, 14, 15, 16, 17]. After the seminal work by the Bochum group [13], Heisenberg and Mihaila employed coupled-cluster theory for structure calculations of ${ }^{16} \mathrm{O}$ based on realistic NN potentials [18.
For their calculation of the charge form factor of ${ }^{16} \mathrm{O}$, they also included selected contributions from 3NFs that could be cast into the form of density-dependent NN interactions [19]. Another recent approach employed $a b$ initio coupled-cluster theory for structure calculations in closed-shell nuclei ${ }^{4} \mathrm{He}$ and ${ }^{16} \mathrm{O}$ 20, 21, 22], in open-shell nuclei as the neighbors of ${ }^{16} \mathrm{O}[23]$, and weakly bound and unbound helium isotopes 24]. These calculations were limited to NN interactions. It is the purpose of this paper to develop coupled-cluster theory for three-body Hamiltonians. This extension of the coupled-cluster method might also find applications in condensed-matter theory and quantum chemistry [25, 26, 27].

A current frontier in nuclear structure theory is to determine consistent 3NFs corresponding to the different $\mathrm{NN}$ interactions, and with predictive power when extrapolated to the extremes of isospin and to moderate densities. Several theoretical approaches are currently being used. The Tucson-Melbourne 3NF already employed symmetries of QCD in its construction [28, 29]. Existing phenomenological 3NFs include the Fujita-Miyazawa force based on $2 \pi$ exchange with an intermediate $\Delta$-isobar 30$]$ and also various shorterranged 3NFs 31, 32, 33, 34]. This approach has led to a very successful description of light nuclei (for a review see Ref. [2]).

Onother approach is to systematically construct NN and higher-order interactions within the framework of chiral effective field theory (EFT) [35, 36, 37, 38]. Chiral interactions are expanded in powers of a typical momentum of nucleons in nuclei and in powers of the pion mass, both generically called $Q$, over the EFT breakdown scale $\Lambda_{\chi} \sim 1 \mathrm{GeV}\left(\approx 5 \mathrm{fm}^{-1}\right)$. The EFT power counting naturally explains the hierarchy of $\mathrm{NN}, 3 \mathrm{~N}$, and higher-body interactions [39], which only enter in subleading orders and make calculations for complex nuclei based on NN and $3 \mathrm{~N}$ interactions meaningful. At this point, the leading $3 \mathrm{NF}$ has been implemented [40, 41, 42]. 
Nuclear interactions require regularization and renormalizaton to be meaningful, and with EFT, usually a momentum cutoff scheme is used [37, 38, 43, 44]. The description of the NN data is not completely independent of the cutoff, but the cutoff variation decreases with increasing order, because the cutoff dependences can be absorbed by higher-order contact interactions. In this way, chiral EFT implements the renormalization group (RG) running of the interactions up to higher-order terms. This is reflected in the leading chiral $3 \mathrm{NF}$, which includes a $3 \mathrm{~N}$ contact term to be fitted to $3 \mathrm{~N}$ data. In principle, nuclear structure calculations based directly on chiral interactions are feasible [7, 45, 46, 47]. However, the typical cutoffs $\left(\Lambda \approx 2.5-3 \mathrm{fm}^{-1}\right)$ are somewhat too large to use chiral interactions without resummations or prediagonalizations in many-body calculations.

Renormalization group methods can be used to evolve nuclear interactions to lower momenta, which leads to improved convergence in few- and many-body calculations [48, 49, 50, 51]. The resulting low-momentum interactions, known generically as " $V_{\text {low }}$ ", have variable momentum cutoffs and are approximately independent of the starting NN interaction for cutoffs $\Lambda \lesssim$ $2 \mathrm{fm}^{-1}$ [48, 49]. The RG evolution preserves the longrange parts, and starting from chiral EFT interactions, generates all higher-order contact operators needed to reproduce low-energy NN observables. Moreover, with increasing orders in EFT, the resulting $V_{\text {low } k}$ interactions are very similar to low-momentum interactions obtained from conventional potentials [49, 52]. Since chiral EFT represents the most general low-momentum expansion of nuclear forces, the above observations motivate combining low-momentum interactions with $3 \mathrm{NFs}$ from chiral EFT [50]. We will follow this approach and employ $V_{\text {low } k}$ with the corresponding low-momentum $3 \mathrm{NF}$ adjusted to the binding energies of ${ }^{3} \mathrm{H}$ and ${ }^{4} \mathrm{He}$ [50]. In this way, we can expect to define approximately consistent NN and $3 \mathrm{~N}$ interactions. In this first study, we focus on the development of the coupled-cluster method to include $3 \mathrm{NFs}$ and will only present results for one cutoff. A study of the cutoff variation and associated uncertainties due to higher-order many-body interactions will be left to future work.

This paper is organized as follows. In Section II we present coupled-cluster theory and its extension to threebody Hamiltonians. In Section III, we solve the resulting coupled-cluster amplitude equations at the singles and doubles (CCSD) level for ${ }^{4} \mathrm{He}$ based on low-momentum $\mathrm{NN}$ and $3 \mathrm{~N}$ interactions. Our findings are especially promising as they show that the binding energy of ${ }^{4} \mathrm{He}$ can be calculated based on those parts of the $3 \mathrm{NF}$ that can be viewed as density-dependent zero-, one-, and twobody forces. We summarize our results in Section IV.

\section{COUPLED-CLUSTER EQUATIONS FOR THREE-NUCLEON FORCES}

This is the main technical section of this paper. In the first subsection, we briefly recapitulate coupled-cluster theory and the reformulation of the three-body Hamiltonian in normal-ordered form. The second subsection deals with the diagrammatic derivation and the factorization of the coupled-cluster equations due to the residual three-body force that remains after normal ordering of the Hamiltonian.

\section{A. Coupled-cluster theory}

We consider a pure three-body Hamiltonian. Coupledcluster theory for one- and two-body Hamiltonians is a mature field, and we refer the reader to the reviews 13 , 14, 15, 16, 17]. The three-body Hamiltonian is written as

$$
\hat{H}_{3}=\frac{1}{36} \sum_{p q r s t u}\langle p q r \| s t u\rangle \hat{a}_{p}^{\dagger} \hat{a}_{q}^{\dagger} \hat{a}_{r}^{\dagger} \hat{a}_{u} \hat{a}_{t} \hat{a}_{s} .
$$

Here, $\langle p q r \| s t u\rangle$ denotes the antisymmetrized three-body matrix elements, while $\hat{a}_{p}^{\dagger}$ and $\hat{a}_{p}$ create and annihilate a fermion in the single-particle orbital $p$, respectively.

In coupled-cluster theory, the Fermi vacuum is a singleparticle product state $|\phi\rangle=\prod_{i=1}^{A} \hat{a}_{i}^{\dagger}|0\rangle$, where the $A$ lowest-energy orbitals are occupied. In a first step, we cast the three-body interaction into a normal-ordered form with respect to this vacuum. In what follows it is assumed that the indices $i, j, k, l, m$ label the occupied orbitals of $|\phi\rangle$ while $a, b, c, d, e$ refer to the unoccupied orbitals of $|\phi\rangle$. The former indices run over the number $n_{o} \equiv A$ of occupied orbitals, while the latter run over the remaining number $n_{u}$ of unoccupied orbitals. Typically, one has $n_{u} \gg n_{o}$. Indices referring to all orbitals are denoted as $p, q, r, s, t, u$; see for example Eq. (1). The normal-ordered Hamiltonian is thus

$$
\begin{aligned}
\hat{H}_{3}= & \frac{1}{6} \sum_{i j k}\langle i j k \| i j k\rangle+\frac{1}{2} \sum_{i j p q}\langle i j p \| i j q\rangle\left\{\hat{a}_{p}^{\dagger} \hat{a}_{q}\right\} \\
& +\frac{1}{4} \sum_{i p q r s}\langle i p q \| i r s\rangle\left\{\hat{a}_{p}^{\dagger} \hat{a}_{q}^{\dagger} \hat{a}_{s} \hat{a}_{r}\right\}+\hat{h}_{3},
\end{aligned}
$$

where $\hat{h}_{3}$ denotes the residual three-body Hamiltonian

$$
\hat{h}_{3} \equiv \frac{1}{36} \sum_{p q r s t u}\langle p q r \| s t u\rangle\left\{\hat{a}_{p}^{\dagger} \hat{a}_{q}^{\dagger} \hat{a}_{r}^{\dagger} \hat{a}_{u} \hat{a}_{t} \hat{a}_{s}\right\} .
$$

Here, we used the $\{\ldots\}$ to denote normal ordering. Writing the three-body Hamiltonian in normal-ordered form, it is clear that the Hamiltonian separates into a zero-, one-, two-, and a three-body term. The first three sums in Eq. (2) are the vacuum expectation value, and the "density-dependent" one- and two-body terms, respectively. Their treatment is standard in coupled-cluster 
theory, as they simply modify the normal-ordered twobody Hamiltonian from the $N N$ interaction. In this section, we focus on the residual three-body operator of Eq. (3).

In coupled-cluster theory, the correlated state $|\psi\rangle$ is given by a correlation operator $\exp (\hat{T})$ that acts onto a single-particle product state $|\phi\rangle=\prod_{i=1}^{A} \hat{a}_{i}^{\dagger}|0\rangle$ of the $A$-body system by

$$
|\psi\rangle=e^{\hat{T}}|\phi\rangle .
$$

The cluster operator,

$$
\hat{T}=\hat{T}_{1}+\hat{T}_{2}+\ldots+\hat{T}_{A},
$$

consists of a one-body cluster operator

$$
\hat{T}_{1}=\sum_{i a} t_{i}^{a} \hat{a}_{a}^{\dagger} \hat{a}_{i}
$$

a two-body cluster operator

$$
\hat{T}_{2}=\frac{1}{4} \sum_{i j a b} t_{i j}^{a b} \hat{a}_{a}^{\dagger} \hat{a}_{b}^{\dagger} \hat{a}_{j} \hat{a}_{i}
$$

and so forth. Note that the two-body cluster amplitudes $t_{i j}^{a b}=-t_{i j}^{b a}=-t_{j i}^{a b}=t_{j i}^{b a}$ are fully antisymmetric. Clearly, $\hat{T}_{1}$ and $\hat{T}_{2}$ induce $1 p-1 h$ and $2 p-2 h$ excitations, respectively. In what follows, we will limit the expansion, Eq. (5), of the cluster operator to the onebody cluster, Eq. (6), and the two-body cluster, Eq. (7), respectively. This approximation is referred to as CCSD ("coupled-cluster theory with single and double excitations"). CCSD is a powerful approximation and a compromise between accuracy on the one hand and computational effort on the other hand. One inserts the ansatz Eq. (4) into the Schrödinger equation, multiplies with $\exp (-\hat{T})$ from the left, and obtains the following set of equations

$$
\begin{aligned}
E & =\langle\phi|\bar{H}| \phi\rangle, \\
0 & =\left\langle\phi_{i}^{a}|\bar{H}| \phi\right\rangle, \\
0 & =\left\langle\phi_{i j}^{a b}|\bar{H}| \phi\right\rangle .
\end{aligned}
$$

Here $\left|\phi_{i_{1} \ldots i_{n}}^{a_{1} \ldots a_{n}}\right\rangle=\hat{a}_{a_{n}}^{\dagger} \ldots \hat{a}_{a_{1}}^{\dagger} \hat{a}_{i_{1}} \ldots \hat{a}_{i_{n}}|\phi\rangle$ is a $n p-n h$ excitation of the product state $|\phi\rangle$, and

$$
\bar{H}=\exp (-\hat{T}) \hat{H} \exp (\hat{T})
$$

is the similarity-transformed Hamiltonian. This Hamiltonian is a sum of one-, two-, and three-body Hamiltonians, i.e. $\hat{H}=\hat{H}_{1}+\hat{H}_{2}+\hat{H}_{3}$. The treatment of this Hamiltonian within coupled-cluster theory is well known, except for the residual three-body term, Eq (3), of the normal-ordered three-body Hamiltonian $\hat{H}_{3}$.

The CCSD Eqs. (9) and (10) determine the oneparticle and two-particle cluster amplitudes $t_{i}^{a}$ and $t_{i j}^{a b}$, respectively. These amplitudes can then be inserted

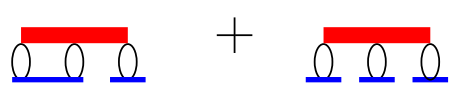

FIG. 1: (Color online) Energy contributions of the residual three-body Hamiltonian, Eq. (3), in the CCSD approximation.

into the first of the CCSD equations, Eq. (8), to determine the ground-state energy. Note that the similaritytransformed Hamiltonian of Eq. (11) is not Hermitian, and CCSD is not a variational approach. However, the similarity-transformed Hamiltonian can be evaluated $e x$ actly for any truncation of the cluster operator. In what follows, we will compute the corrections to the energy, Eq. (8), and to the CCSD Eqs. (9) and (10), that arise due to the residual three-body Hamiltonian.

\section{B. Derivation of coupled-cluster equations}

In this subsection, we derive the contribution of the residual three-body term $\hat{h}_{3}$ from Eq. (3) to the energy, Eq. (8), and the cluster amplitudes, Eqs. (9) and (10). The final results are given in Eq. (12) for the energy and in Eqs. (15) and (16) for the cluster amplitudes, respectively. The contributions to the energy and the coupledcluster amplitudes can be derived, for instance, by following the approach of Ref. [16]. The resulting expressions contain 2, 15 and 51 terms for the energy and the cluster amplitudes. Many of the individual terms consist of sub-terms of similar structure. The theoretical derivation presented in this subsection exploits this structure and leads to an efficient numerical implementation.

It is most convenient to evaluate the matrix elements in Eq. (8) of the similarity-transformed Hamiltonian, Eq. (11), in a diagrammatic form (see, for example, [16, [53]). Diagrams are a useful book keeping device to keep track of the (considerable) number of possible Wick contractions. We refer the reader to the literature for a more detailed description.

In a first step, we determine the correction to the CCSD energy, Eq. (8), that is due to the residual threebody Hamiltonian (3). The matrix element $\left\langle\phi\left|\overline{h_{3}}\right| \phi\right\rangle$ is a sum of all topologically different diagrams, where the Hamiltonian, Eq. (3), is fully contracted by the cluster operators. The two diagrams that enter this expression are presented in Fig. 11 In these diagrams, the thick horizontal bar represents the residual three-body Hamiltonian, Eq. (3); the thin horizontal bars denote the onebody and two-body cluster operators, respectively. Particle and hole lines are also shown. If uncontracted, the former have an arrow that points upward while the latter have an arrow that points downward. The corresponding 


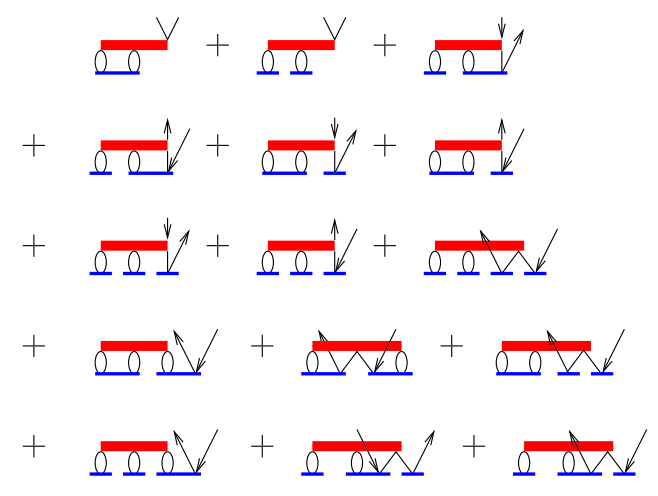

FIG. 2: (Color online) Contributions of the three-body Hamiltonian, Eq. (3), to the $\hat{T}_{1}$ cluster equation in the CCSD approximation.

algebraic expression for the energy correction is

$$
\begin{aligned}
e_{3} & =\left\langle\phi\left|\left(\hat{H} \hat{T}_{1} \hat{T}_{2}\right)_{c}\right| \phi\right\rangle+\left\langle\phi\left|\left(\frac{1}{6} \hat{H} \hat{T}_{1}^{3}\right)_{c}\right| \phi\right\rangle, \\
& =\frac{1}{4} \sum_{k l m c d e}\langle k l m \| c d e\rangle t_{k}^{c} t_{l m}^{d e} \\
& +\frac{1}{6} \sum_{k l m c d e}\langle k l m \| c d e\rangle t_{k}^{c} t_{l}^{d} t_{m}^{e} .
\end{aligned}
$$

This is the energy correction due to the residual threebody Hamiltonian. Note that the computational effort of the energy, Eq. (12), scales as $O\left(n_{u}^{3} n_{o}^{3}\right)$. Note also that the summation over $\sum_{k c} t_{k}^{c}$ is common to both diagrams and might therefore be factored out. This is the basic idea behind the factorization [53], and will be presented in detail below.

Let us consider the matrix element $\left\langle\phi_{i}^{a}|\bar{H}| \phi\right\rangle$ appearing in the CCSD Eq. (9). It is given by the sum over all topologically different diagrams where one particle line and one hole line are not contracted. Figure 2 shows the 15 diagrams that enter this matrix element.

The translation of these diagrams into algebraic expressions is straightforward, but there are two reasons to not present them here. First, the naive numerical implementation of the resulting expressions would be inefficient. To see this, consider, for instance, the third diagram in Fig. 2. The corresponding algebraic expression is

$$
-\frac{1}{2} \sum_{c k d l m}\langle k l m \| c d i\rangle t_{k}^{c} t_{l m}^{d a}
$$

and its naive evaluation costs $O\left(n_{u}^{3} n_{o}^{4}\right)$ operations. However, performing the summations involving the $\hat{T}_{1}$ cluster operator first yields the intermediate

$$
I_{d i}^{l m} \equiv \frac{1}{2} \sum_{c k}\langle k l m \| c d i\rangle t_{k}^{c}
$$

which only costs $O\left(n_{u}^{2} n_{o}^{4}\right)$ operations and requires $O\left(n_{u} n_{o}^{3}\right)$ in memory. The subsequent contraction of this intermediate with the remaining $\hat{T}_{2}$ cluster operator only costs $O\left(n_{u}^{2} n_{o}^{3}\right)$ operations. Clearly, the memory cost of the intermediate is overcompensated by the reduction of computational cycles. Note also that the intermediate Eq. (14) enters in the evaluation of the seventh diagram depicted in Fig. 2, The second reason is that the complexity of the involved diagrams increases rapidly. The number of diagrams increases from 2 to 15 to 51, when going from Eq. (8) to Eq. (9) to Eq. (10), respectively. The construction of each individual diagram and its inspection regarding the construction and use of intermediates then becomes cumbersome, and a more systematic approach is called for. Similar comments apply when improving the coupled-cluster wave function through the inclusion of three-body or four-body cluster amplitudes. One therefore considers a factorization of the coupledcluster equations [53]. This approach yields a very compact form of the coupled-cluster equations and is particularly useful for the numerical implementation [54, 55, , 56] . So far, factorized coupled-cluster equations have been derived in a two-step procedure. The first step consists of constructing all topologically different coupled-cluster diagrams. In a second step, these diagrams are analyzed and repeatedly decomposed into simpler intermediates that undergo single contractions. Here, we proceed differently and present a direct diagrammatic derivation of the factorized coupled-cluster equations. Our derivation avoids the explicit construction of all individual coupledcluster diagrams.

We have to decide in which order multiple contractions of the Hamiltonian with the cluster operators should be performed. Let $T_{k}\left(p^{n} h^{m}\right)$ denote the contraction of the Hamiltonian with the $k$-body cluster operator $\hat{T}_{k}$ via $n$ particle lines and $m$ hole lines. The contraction of $T_{k}\left(p^{n} h^{m}\right)$ with an object of $i$ particle lines and $j$ hole lines costs $c n_{u}^{i} n_{o}^{j}$ computational operations and results in an object of size $m n_{u}^{i} n_{o}^{j}$. Here, $c$ and $m$ denote the computational cost and memory multiplier, respectively, and one finds

$$
\begin{aligned}
c & =n_{u}^{k-n} n_{o}^{k-m}, \\
m & =n_{u}^{k-2 n} n_{o}^{k-2 m} .
\end{aligned}
$$

Based on this analysis, we find that the cost of two subsequent contractions labeled $T_{A}$ and $T_{B}$, respectively, is proportional to $c\left(T_{A}\right)+m\left(T_{A}\right) c\left(T_{B}\right)$ when contraction $T_{A}$ is first, and proportional to $c\left(T_{B}\right)+m\left(T_{B}\right) c\left(T_{A}\right)$ when contraction $T_{B}$ is first. Table \) shows (from left to right) the optimal order in which subsequent contractions should be performed, under the condition that $1 \ll n_{o} \ll n_{u}$ and $n_{o}^{2}<n_{u}$. We also listed the relative computational cost $c$, and the memory multiplier $m$. For the first 7 entries, the order is easily understood. These contractions do not yield an increase of the size of the contracted object (since $m \leqslant 1$ ), and the order is therefore determined by the computational cost. The remaining four contractions increase the size of the contracted object (since $m>1$ ), and it is usually most efficient to 
TABLE I: Memory multiplication factor $m$ and computational cost factor $c$ for forming intermediates through contraction with the $k$-body cluster operator $T_{k}\left(p^{n} h^{m}\right)$ via $n$ particle lines and $m$ hole lines.

\begin{tabular}{|c|c|c|c|c|c|c|c|c|c|c|c|}
\hline & $T_{2}\left(p^{2} h^{2}\right)$ & $T_{1}(p h)$ & $T_{2}\left(p^{2} h\right)$ & $T_{1}(p)$ & $T_{2}\left(p^{2}\right)$ & $T_{2}\left(p h^{2}\right)$ & $T_{2}(p h)$ & $T_{2}(p)$ & $T_{2}\left(h^{2}\right)$ & $T_{1}(h)$ & $T_{2}(h)$ \\
\hline$m$ & $\left(n_{u} n_{o}\right)^{-2}$ & $\left(n_{u} n_{o}\right)^{-1}$ & $n_{u}^{-2}$ & $n_{o} / n_{u}$ & $\left(n_{o} / n_{u}\right)^{2}$ & $n_{o}^{-2}$ & 1 & $n_{o}^{2}$ & $\left(n_{u} / n_{o}\right)^{2}$ & $n_{u} / n_{o}$ & $n_{u}^{2}$ \\
\hline$c$ & 1 & 1 & $n_{o}$ & $n_{o}$ & $n_{o}^{2}$ & $n_{u}$ & $n_{u} n_{o}$ & $n_{u} n_{o}^{2}$ & $n_{u}^{2}$ & $n_{u}$ & $n_{u}^{2} n_{o}$ \\
\hline
\end{tabular}

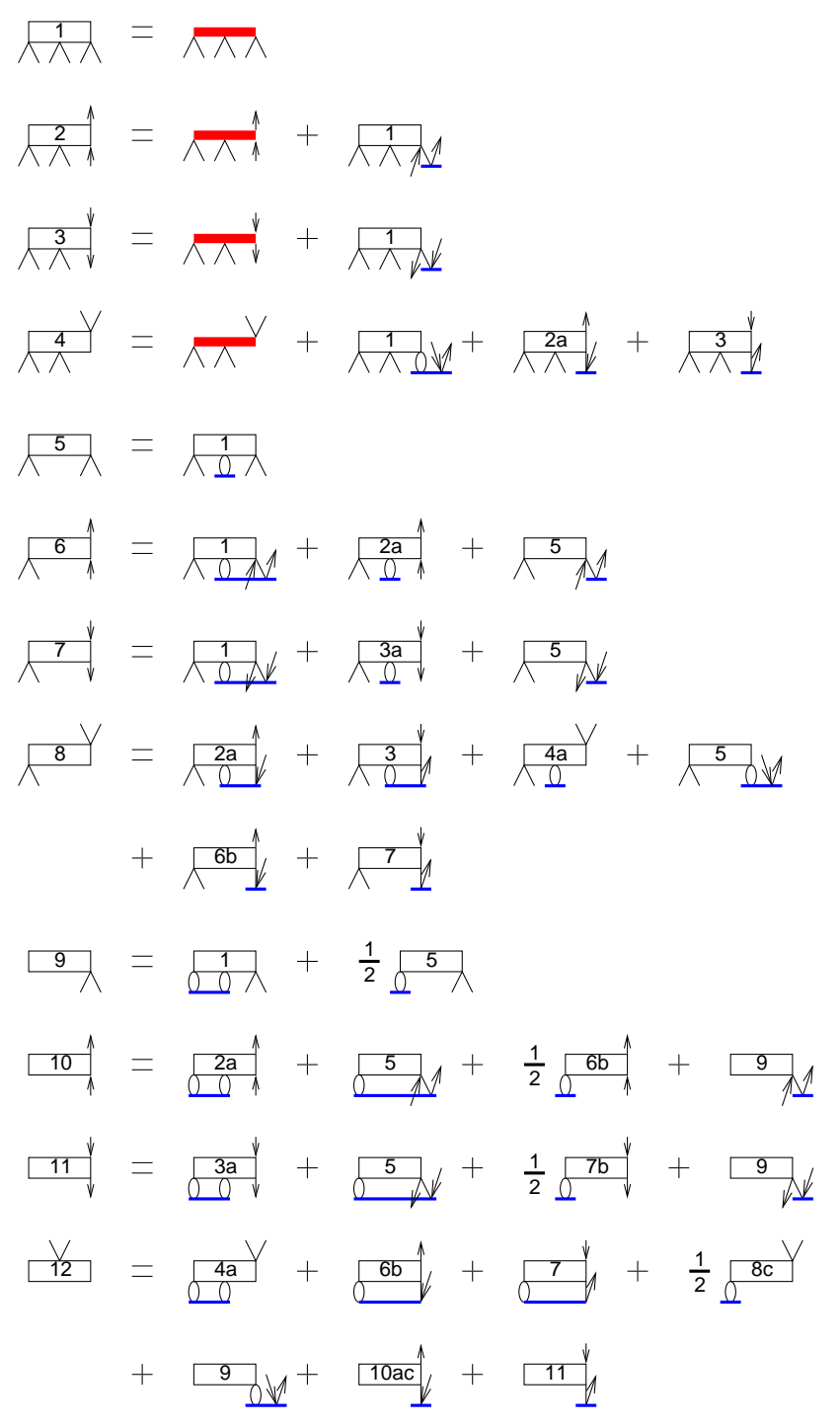

FIG. 3: (Color online) Matrix elements of the residual three-body part of the similarity-transformed Hamiltonian, Eq. (11), that enter the amplitude Eq. (9).

perform the computationally more expensive contraction before performing the second contraction on an object with increased size.

Let us now turn to the diagrammatic factorization of the coupled-cluster equations. Considering Eq. (91) and Fig. 2. we have to construct all coupled-cluster diagrams with one incoming hole line and one outgoing particle line. These diagrams should be constructed from simpler diagrams, adding one contraction at each step. For the residual three-body Hamiltonian, the root is clearly given by the diagram 1 in Fig. 3. This diagram is the only one that has three incoming particle lines and three outgoing hole lines. Diagrams 2 and 3 have one outgoing particle line and one incoming hole line, respectively, and also have a total of six outgoing and incoming lines. They are sums of two diagrams. The first is the residual threebody Hamiltonian with this appropriate particle and hole lines, while the second diagram is a contraction of diagram 1 with a $\hat{T}_{1}$ cluster operator.

In what follows, we adopt the following convention. For a diagram that is the sum of diagrams, we label the first, second, third, etc. term of the sum by a, b, c, respectively. For example, diagram 2 is the sum of diagram $2 \mathrm{a}$ and diagram $2 \mathrm{~b}$. These labels are not printed in Fig. 3. Diagram 4 is the sum of 4 diagrams, namely the corresponding residual three-body Hamiltonian and three contractions of previously generated diagrams. Note that out of the two diagrams of diagram 2 , only diagram $2 \mathrm{a}$ enters. This is due to the order specified in Table \ Diagram $2 \mathrm{~b}$ is a contraction of diagram 1 with $T_{1}(p)$, and this contraction cannot be followed by the contraction $T_{1}(p)$. It is now clear how to proceed. Diagrams 5 to 8 have a total of four incoming and outgoing lines, while diagrams 9 to 12 have a total of two incoming and outgoing lines, respectively. Note the peculiar factor $1 / 2$ in front of diagram $9 \mathrm{~b}$. This diagram is a $T_{1}(p h)$ contraction of diagram $5 \mathrm{~b}$ which itself is also a $T_{1}(p h)$ contraction. The factor $1 / 2$ will be needed for the translation into algebraic expressions. Similar comments apply to diagrams $10 \mathrm{c}, 11 \mathrm{c}$, and $12 \mathrm{~d}$. Note that diagram 12 consists of all diagrams with one incoming hole line and one outgoing particle line. The recursive expansion of the corresponding right-hand side yields indeed all diagrams depicted in Fig. 2], and therefore factors the CCSD Eq. (9).

Let us translate Fig. 2 into algebraic expressions. We use the convention that the intermediate $I_{p_{1}, p_{2}, \ldots}^{q_{1}, q_{2}, \ldots}$ has incoming lines $p_{1}, p_{2}, \ldots$ and outgoing lines $q_{1}, q_{2}, \ldots$, respectively. In what follows, the intermediate $I(\nu)$ corresponds to diagram $\nu$ of Fig. 3. We restrict ourselves to those intermediates that are needed for the construction of diagram 12, and denote the diagrams 1, 2a, 3a, and 4a directly in terms of the corresponding three-body matrix 


$$
\begin{aligned}
& \left.\right|_{1 \wedge 1} ^{13}=\hat{\imath \wedge \hat{\imath}}
\end{aligned}
$$

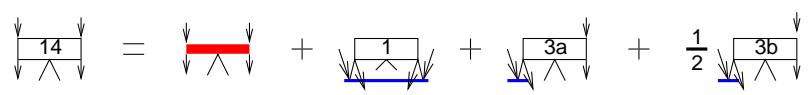

$$
\begin{aligned}
& \sqrt{151}=\sqrt{151}
\end{aligned}
$$

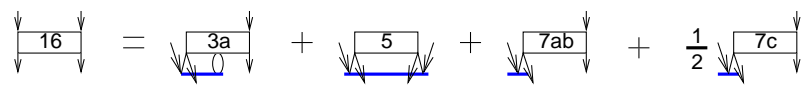

$$
\begin{aligned}
& +\frac{k 14 a^{k}}{\sqrt{14}} \\
& \hat{117}_{1 \wedge}=\hat{\Lambda \wedge}
\end{aligned}
$$

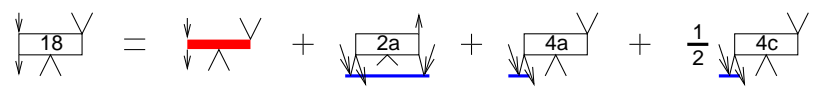

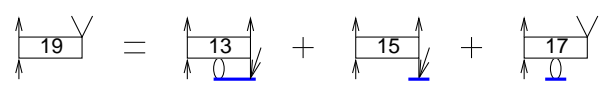

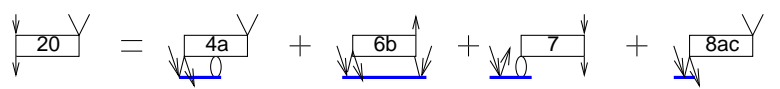

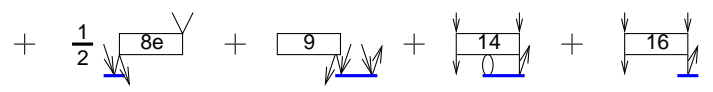

$$
\begin{aligned}
& +\frac{k 18 \mathrm{a} Y}{\sqrt{2}} \\
& \frac{V_{21} Y}{\wedge}=\frac{V_{\Lambda}}{\wedge}
\end{aligned}
$$

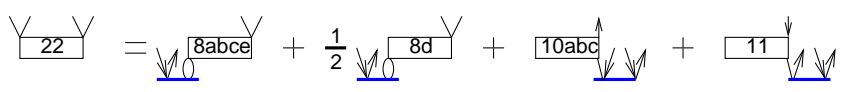

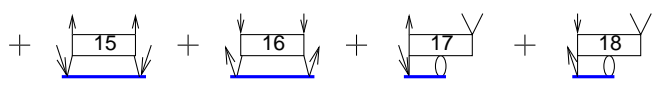

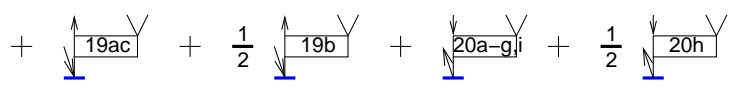

$$
\begin{aligned}
& +\frac{Y_{21} Y}{0}
\end{aligned}
$$

FIG. 4: (Color online) Additional diagrams needed for the contributions of the residual three-body Hamiltonian, Eq. (3), to the CCSD Eq. (10).

elements. The result is

$$
\begin{aligned}
I(5)_{c e}^{k m} & =\frac{1}{4} \sum_{d l}\langle k l m \| c d e\rangle t_{l}^{d}, \\
I(6 \mathrm{~b})_{c e}^{k a} & =\frac{1}{2} \sum_{d l}\langle k l a \| c d e\rangle t_{l}^{d}, \\
I(7 \mathrm{~b})_{c i}^{k m} & =\frac{1}{2} \sum_{d l}\langle k l m \| c d i\rangle t_{l}^{d}, \\
I(7)_{c i}^{k m} & =\frac{1}{4} \sum_{l d e}\langle k l m \| c d e\rangle t_{l i}^{d e}+I(7 \mathrm{~b})_{c i}^{k m} \\
& +2 \sum_{e} I(5)_{c e}^{k m} t_{i}^{e}, \\
I(8 \mathrm{c})_{c i}^{k a} & =\sum_{d l}\langle k l a \| c d i\rangle t_{l}^{d},
\end{aligned}
$$

and

$$
\begin{aligned}
I(10 \mathrm{ac})_{e}^{a} & =\frac{1}{4} \sum_{c k d l}\langle k l a \| c d e\rangle t_{k l}^{c d}+\sum_{k c} I(6 \mathrm{~b})_{c e}^{k a} t_{k}^{c}, \\
I(11)_{i}^{m} & =\frac{1}{4} \sum_{c k d l}\langle k l m \| c d i\rangle t_{k l}^{c d}+2 \sum_{k c e} I(5)_{c e}^{k m} t_{k i}^{c e} \\
& +\sum_{k c} I(7 \mathrm{~b})_{c i}^{k m} t_{k}^{c}+\sum_{c} I(9)_{c}^{m} t_{i}^{c} .
\end{aligned}
$$

In terms of these intermediates, the correction to the 
right hand side of the CCSD Eq. (9) reads

$$
\begin{aligned}
& \frac{1}{4} \sum_{c k d l}\langle k l a \| c d i\rangle t_{k l}^{c d}+\sum_{c k e} I(6 \mathrm{~b})_{c e}^{k a} t_{k i}^{c e} \\
- & \sum_{c k m} I(7)_{c i}^{k m} t_{k m}^{c a}+\frac{1}{2} \sum_{k c} I(8 \mathrm{c})_{c i}^{k a} t_{k}^{c}+\sum_{c k} I(9)_{c}^{k} t_{k i}^{c a} \\
+ & \sum_{e} I(10 \mathrm{ac})_{e}^{a} t_{i}^{e}-\sum_{m} I(11)_{i}^{m} t_{m}^{a} .
\end{aligned}
$$

These terms have to be added to the well-known CCSD equation for the $T_{1}$-cluster amplitudes based on two-body Hamiltonians.

For the factorization of the CCSD Eq. (10), one has to construct all diagrams with two incoming hole lines and two outgoing particle lines. Their number is 51 , and we directly derive them in a factorized form. Figure 4 shows the factorized diagrams that are needed for the recursive construction of the CCSD Eq. (10). The corresponding algebraic expressions for the intermediates are

$$
\begin{aligned}
& I(3 b)_{c d i}^{k l m}=\frac{1}{12} \sum_{e}\langle k \operatorname{lm} \| c d e\rangle t_{i}^{e}, \\
& I(3)_{c d i}^{k l m}=\frac{1}{12}\langle k l m \| c d i\rangle+I(3 b)_{c d i}^{k l m}, \\
& I(4 c)_{c d i}^{k l a}=\frac{1}{4} \sum_{e}\langle k l a \| c d e\rangle t_{i}^{e}, \\
& I(7 c)_{c i}^{k m}=2 \sum_{e} I(5)_{c e}^{k m} t_{i}^{e}, \\
& I(7 a b)_{c i}^{k m}=I(7)_{c i}^{k m}-I(7 c)_{c i}^{k m}, \\
& I(8 a c)_{c i}^{k a}=\frac{1}{2} \sum_{l d e}\langle k l a \| c d e\rangle t_{l i}^{d e}+I(8 c)_{c i}^{k a}, \\
& I(8 b)_{c i}^{k a}=-6 \sum_{l m d} I(3)_{c d i}^{k l m} t_{l m}^{d a}, \\
& I(8 d)_{c i}^{k a}=4 \sum_{m e} I(5)_{c e}^{k m} t_{m i}^{e a}, \\
& I(8 e)_{c i}^{k a}=2 \sum_{e} I(6 b)_{c e}^{k a} t_{i}^{e}, \\
& I(8 a b c e)_{c i}^{k a}=I(8 a c)_{c i}^{k a}+I(8 b)_{c i}^{k a}+I(8 e)_{c i}^{k a} \text {, } \\
& I(10 a b c)_{e}^{a}=I(10 a c)_{e}^{a}-2 \sum_{k m c} I(5)_{c e}^{k m} t_{k m}^{c a}, \\
& I(14)_{i d j}^{k l m}=\frac{1}{12}\langle k l m \| i d j\rangle+\frac{1}{24} \sum_{c e}\langle k l m \| c d e\rangle t_{i j}^{c e} \\
& +\sum_{c}\left(\frac{1}{6}\langle k l m \| c d j\rangle+I(3 b)_{c d j}^{k l m}\right) t_{i}^{c},
\end{aligned}
$$

and

$$
\begin{aligned}
I(15)_{c e}^{a b} & =\frac{1}{2} \sum_{l d}\langle a l b \| c d e\rangle t_{l}^{d}, \\
I(16)_{i j}^{k m} & =\frac{1}{4} \sum_{c d l}\langle k l m \| c d j\rangle t_{l i}^{d c}+\frac{1}{2} \sum_{c e} I(5)_{c e}^{k m} t_{i j}^{c e} \\
& +\sum_{c}\left(I(7 a b)_{c j}^{k m}+\frac{1}{2} I(7 c)_{c j}^{k m}\right) t_{i}^{c} \\
& +\frac{1}{4} \sum_{l d}\langle k l m \| i d j\rangle t_{l}^{d}, \\
I(18)_{i d j}^{k l b} & =\frac{1}{4}\langle k l b \| i d j\rangle+\frac{1}{8} \sum_{c e}\langle k l b \| c d e\rangle t_{i j}^{c e} \\
& +\sum_{c}\left(\frac{1}{2}\langle k l b \| c d j\rangle+I(4 c)_{c d j}^{k l b}\right) t_{i}^{c}, \\
I(19 a c)_{c j}^{a b} & =\frac{1}{2} \sum_{l d e}\langle a l b \| c d e\rangle t_{l j}^{d e}+\sum_{l d}\langle a l b \| c d j\rangle t_{l}^{d}, \\
I(19 b)_{c j}^{a b} & =2 \sum_{e} I(15)_{c e}^{a b} t_{j}^{e}, \\
I(20 a-g, i)_{i j}^{k b} & =\frac{1}{2} \sum_{c l d}\langle k l b \| c d j\rangle t_{i l}^{c d}+\frac{1}{2} \sum_{e c} I(6 b)_{c e}^{k b} t_{i j}^{c e} \\
& +2 \sum_{m c} I(7)_{c i}^{m k} t_{m j}^{c b}+\frac{1}{2} \sum_{l d}\langle k l b \| i d j\rangle t_{l}^{d} \\
& +\sum_{c}\left(I(8 a c)_{c j}^{k b}+I(8 e)_{c j}^{k b}\right) t_{i}^{c} \\
& +\frac{1}{2} \sum_{c} I(9)_{c}^{k} t_{i j}^{c b}-3 \sum_{l d m} I(14)_{i d j}^{k l m} t_{l m}^{d b}, \\
& -2 \sum_{m} I(16)_{i j}^{k m} t_{m}^{b} . \\
k(20 h & \\
k b &
\end{aligned}
$$

The residual three-body Hamiltonian, Eq. (3), thus leads to the following correction, to be added to the right hand side of the CCSD Eq. (10), 


$$
\begin{aligned}
& \sum_{l d}\langle a l b \| i d j\rangle t_{l}^{d}+\frac{1}{2} P(i j) \sum_{l c d}\langle a l b \| c d j\rangle t_{l i}^{d c}+P(a b) P(i j) \sum_{k c}\left(I(8 a b c e)_{c i}^{k a}+\frac{1}{2} I(8 d)_{c i}^{k a}\right) t_{k j}^{c b} \\
+ & P(a b) \sum_{e} I(10 a b c)_{e}^{a} t_{i j}^{e b}-P(i j) \sum_{m} I(11)_{i}^{m} t_{m j}^{a b}+\sum_{c e} I(15)_{c e}^{a b} c_{i j}^{c e}+P(i j) \sum_{k m} I(16)_{i j}^{k m} t_{k m}^{a b} \\
- & P(a b) P(i j) \sum_{k l d} I(18)_{i d j}^{k l b} t_{l k}^{d a}+P(i j) \sum_{c}\left(I(19 a c)_{c j}^{a b}+\frac{1}{2} I(19 b)_{c j}^{a b}\right) t_{i}^{c} \\
- & P(a b) P(i j) \sum_{k}\left(I(20 a-g, i)_{i j}^{k b}+\frac{1}{2} I(20 h)_{i j}^{k b}\right) t_{k}^{a} .
\end{aligned}
$$

Here the permutation $P(a b)$ implies $P(a b) I_{a b}=I_{a b}-I_{b a}$. Again, these have to be added to the right hand side of the CCSD Eq. (10). Equation (12) for the energy correction, and the expressions of Eqs. (15) and (16) for the cluster amplitudes are the main technical results of this paper. Upon expansion of diagram 22 in Fig. 4. one gets indeed all 51 diagrams that enter the coupled-cluster Eq. (10). The numerical implementation of the terms in Eqs. (15) and (16) is straightforward. It is interesting to analyze the resulting computational costs. The most expensive intermediates $I(18)$ and $I(19 a c)$ cost $n_{u}^{4} n_{o}^{4}$ and $n_{u}^{5} n_{o}^{2}$ computational cycles, respectively. The most memory-expensive object is the interaction $\langle a l b|| c d e\rangle$ which enters the construction of intermediates $I(15)$ and $I(19 a c)$, and requires the storage of $n_{u}^{5} n_{o}$ real numbers. In our largest calculations for ${ }^{4} \mathrm{He}$, we have $n_{o}=4$ and $n_{u}=220$.

\section{APPLICATION TO ${ }^{4} \mathrm{He}$}

We present the first ab-initio coupled-cluster calculations including 3NFs. Our results are based on lowmomentum $\mathrm{NN}$ [49] and 3N [50] interactions,

$$
H=T+V_{\text {low } k}(\Lambda)+V_{3 \mathrm{~N}}(\Lambda) .
$$

In this exploratory study we use a sharp cutoff $\Lambda=$ $1.9 \mathrm{fm}^{-1}$, and $V_{\text {low } k}$ is derived from the Argonne $v_{18}$ potential [57]. The corresponding $3 \mathrm{~N}$ interaction is based on the leading chiral $3 \mathrm{NF}$ and has been fitted to the ${ }^{3} \mathrm{H}$ and ${ }^{4} \mathrm{He}$ binding energies in Ref. [50]. This 3NF consists of a long-range $2 \pi$-exchange part, determined by the lowenergy coefficients $c_{1}, c_{3}$, and $c_{4}$, an intermediate-range $1 \pi$-exchange (" $D$-term") and a short-range contact interaction ("E-term") [40, 42]. The 3NF operators are multiplied by regulating functions of the incoming and outgoing Jacobi momenta, $f_{\mathrm{R}}(p, q)=\exp \left[-\left(\left(p^{2}+\frac{3 q^{2}}{4}\right) / \Lambda^{2}\right)^{4}\right]$, with the same cutoff value $\Lambda$ as in $V_{\text {low } k}$. For additional details on the $3 \mathrm{NF}$, we refer the reader to Refs. [50, 51]. These low-momentum interactions can be directly employed within coupled-cluster theory.

In this application of coupled-cluster theory we restrict ourselves to a proof-of-principle calculation. For

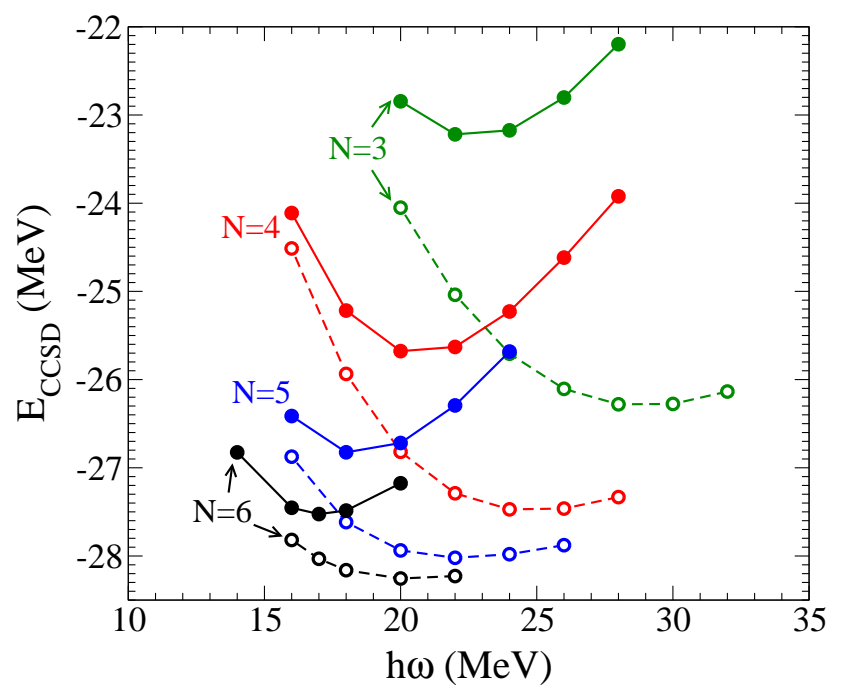

FIG. 5: (Color online) CCSD results for the binding energy of ${ }^{4} \mathrm{He}$ as a function of the oscillator spacing and for model spaces consisting of $N=3$ to $N=6$ oscillator shells. The CCSD calculations are based on low-momentum NN and $3 \mathrm{~N}$ interactions, where the full and dashed lines respectively denote the energy obtained with and without 3NFs.

simplicity, we have therefore considered only the $3 \mathrm{NF}$ channel with total isospin $T=1 / 2$, total angular momentum $J=1 / 2$, and positive parity. This partial wave is the dominant contribution to the binding energies of light nuclei: For ${ }^{4} \mathrm{He}$, the corresponding FaddeevYakubovsky result is $E=-28.20(5) \mathrm{MeV}$, which differs only by $100 \mathrm{keV}$ from the exact energy including all partial waves, $E=-28.30(5) \mathrm{MeV}[50]$.

The coupled-cluster calculations are performed in a harmonic oscillator (HO) basis, with basis parameters given by the oscillator spacing $\hbar \omega$ and the number $N$ of oscillator shells. We will present our results as a function of these parameters. The matrix elements of the $3 \mathrm{NF}$ are calculated first in relative $\mathrm{HO}$ states given by the expansion in $3 \mathrm{~N}$ partial waves. For the transformation from relative $\mathrm{HO}$ states to the single-particle (" $m$-scheme") basis, we essentially follow the Appendix of Ref. [7]. However, we can also start from a $3 \mathrm{NF}$ in 


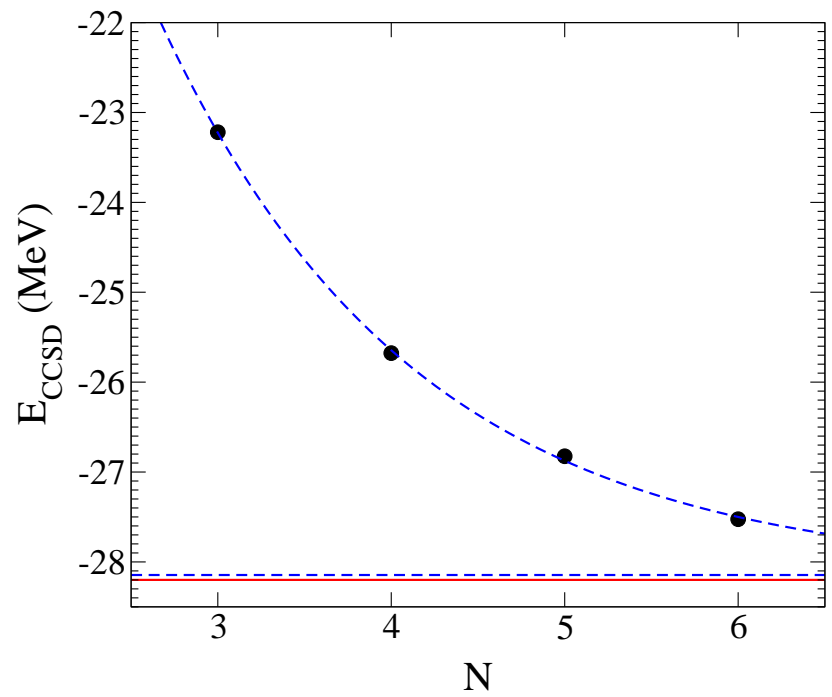

FIG. 6: (Color online) Data points: CCSD results (taken at the $\hbar \omega$ minima) for the binding energy of ${ }^{4} \mathrm{He}$ with $3 \mathrm{NFs}$ as a function of the number of oscillator shells. Dashed lines: Exponential fit to the data and asymptote of the fit. Full line: Exact result.

the non-antisymmetrized HO basis, which simplifies the transformations given in Eqs. (B.9), (B.11), and (B.12) of Ref. 7], and explicitly antisymmetrize the $m$-scheme matrix elements in the last step of our transformation. This approach does not require coefficients of fractional parentage. We verified that our matrix elements agree with those obtained from a transformation based on antisymmetrized relative $\mathrm{HO}$ matrix elements. We further have checked that the transformation preserves the unit matrix and that it yields identical matrix elements for $\boldsymbol{\sigma}_{1} \cdot \boldsymbol{\sigma}_{2}$ and $\boldsymbol{\sigma}_{2} \cdot \boldsymbol{\sigma}_{3}$ in antisymmetrized states.

In Fig. 5, our CCSD results for the binding energy of ${ }^{4} \mathrm{He}$ are shown with and without $3 \mathrm{NFs}$ as a function of the oscillator spacing and with increasing model space size. The 3NF contribution is repulsive, in agreement with the Faddeev-Yakubovsky calculation [50], and correspondingly, the minima in $\hbar \omega$ are shifted to smaller oscillator spacings. We observe a slow convergence at the last few $100 \mathrm{keV}$ level, which is due to the sharp cutoff in $V_{\text {low } k}$. This might be improved by using low-momentum interactions with smooth cutoffs [58]. Using the minima of the CCSD results with 3NFs, we make an exponential fit of the form $E(N)=E_{\infty}+a \exp (-b N)$ to the data points. The result is shown in Fig. 6 The extrapolated infinite model space value is $E_{\infty}=-28.09 \mathrm{MeV}$, which is very close to the exact result $E=-28.20(5) \mathrm{MeV}$.

It is interesting to analyze the different contributions $\Delta E$ to the binding energy $E$. The individual contributions are given in Fig. 7 for a model space of $N=4$ oscillator shells and $\hbar \omega=20 \mathrm{MeV}$. The main contribution stems from the low-momentum NN interaction. The contributions from 3NFs account only for about $10 \%$ of the total binding energy. This is consistent with the chiral EFT power-counting estimate

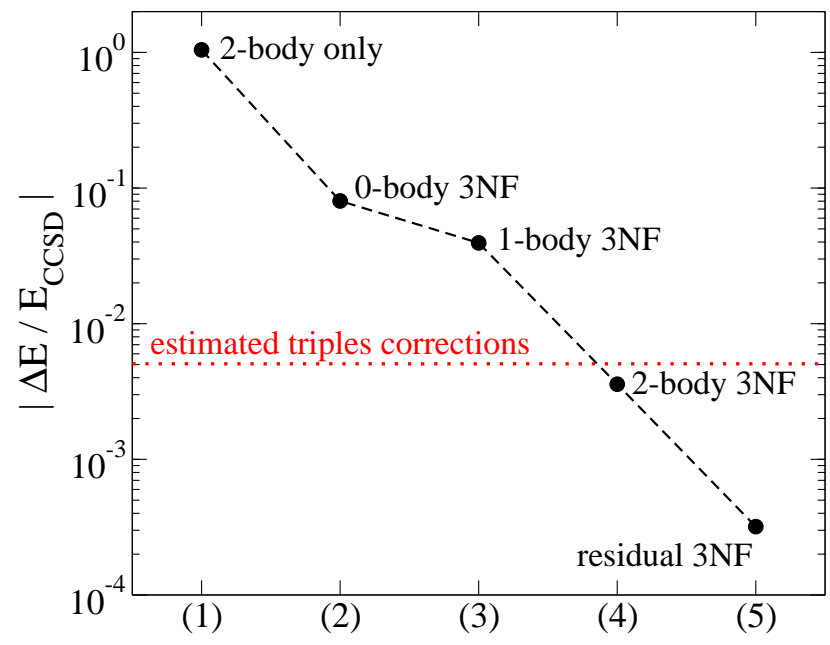

FIG. 7: (Color online) Relative contributions $|\Delta E / E|$ to the binding energy of ${ }^{4} \mathrm{He}$ at the CCSD level. The different points denote the contributions from (1) low-momentum NN interactions, (2) the vacuum expectation value of the $3 \mathrm{NF},(3)$ the normal-ordered one-body Hamiltonian due to the $3 \mathrm{NF}$, (4) the normal-ordered two-body Hamiltonian due to the 3NF, and (5) the residual 3NFs. The dotted line estimates the corrections due to omitted three-particle-three-hole clusters.

$\left\langle V_{3 \mathrm{~N}}\right\rangle \sim\left(Q / \Lambda_{\chi}\right)^{3}\left\langle V_{\text {low } k}\right\rangle \approx 0.1\left\langle V_{\text {low } k}\right\rangle$ [50] (see also Table 1 in Ref. [52]). The second, third, and fourth largest contribution are due to the first, second, and third term on the right-hand side of Eq. (2). These are the density-dependent zero-, one-, and two-body terms, which resulted from the normal ordering of the three-body Hamiltonian in coupled-cluster theory. The contributions from the residual three-body Hamiltonian, Eq. (3), are very small and are represented by the last point in Fig. 7. Recall that the residual 3NF contributes to the energy directly through Eq. (12) and indirectly through a modification of the cluster amplitudes via Eqs. (15) and (16). Apparently, both contributions are very small. In addition and independent of the result that low-momentum $3 \mathrm{~N}$ interactions are perturbative for cutoffs $\Lambda \lesssim 2 \mathrm{fm}^{-1}$ [50], we find here that the contributions of $3 \mathrm{NFs}$ decrease rapidly with increasing rank of the normal-ordered terms.

The small contribution from the residual three-body Hamiltonian is the most important result of our study. It suggests that one can neglect the residual terms of the $3 \mathrm{NF}$ when computing binding energies of light nuclei. This is not unexpected and has been anticipated in several earlier studies. Mihaila and Heisenberg [19] computed the charge form factor for ${ }^{16} \mathrm{O}$ within coupled-cluster theory and found a very good agreement with experimental data by considering only the densitydependent one- and two-body parts of 3NFs. Similarly, Navrátil and Ormand [59] observed in no-core shellmodel calculations that density-dependent two-body terms are the most significant contributions of effective three-body forces. Our finding also support Zuker's 60] 


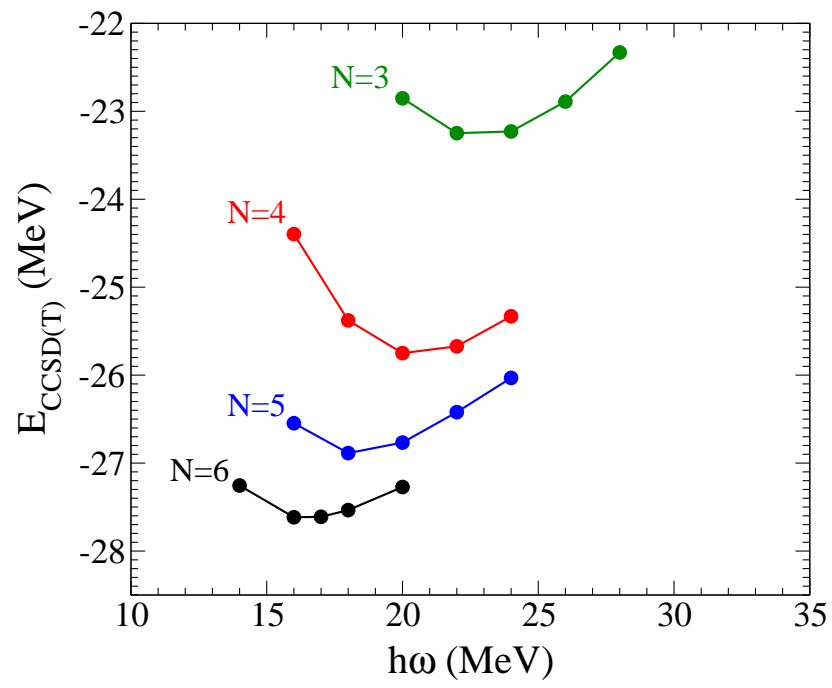

FIG. 8: (Color online) CCSD(T) results for the binding energy of ${ }^{4} \mathrm{He}$ as a function of the oscillator spacing and for model spaces consisting of $N=3$ to $N=6$ oscillator shells. The contributions from $3 \mathrm{NFs}$ are limited to the densitydependent zero-, one-, and two-body terms and exclude its residual three-body terms.

idea that monopole corrections to valence-shell interactions are due to the density-dependent terms of 3NFs. Note finally that the modeling of three-body interactions in terms of density-dependent two-body Hamiltonians has a long history, see e.g. Ref. [61]. Note that all these examples and the present study employ sufficiently "soft" or "effective" interactions. We expect that the smallness of residual 3NFs is a property of such interactions. We will study the cutoff dependence of this finding in future work. Finally, the smallness of residual 3NFs is also encouraging for future improved nuclear matter calculations, which currently include low-momentum 3NFs through density-dependent NN interactions [51].

The smallness of the residual three-body terms is also for coupled-cluster calculations a most welcome result. This is attractive for two reasons. First, the inclusion of the residual three-nucleon Hamiltonian, as described in Subsection IIB, is computationally expensive. It exceeds the cost of a CCSD calculation for two-body Hamiltonians by a factor of order $O\left(n_{u}\right)+O\left(n_{o}^{2}\right)$ and is therefore significant for a large number of unoccupied orbitals and/or large number of nucleons. Second, the omission of the residual three-body Hamiltonian will allow us to treat $3 \mathrm{NFs}$ within the standard coupled-cluster theory developed for two-body Hamiltonians (after normal ordering). As a result, we can take the CCSD calculations one step further and include perturbative corrections of three-particle-three-hole clusters [62].

Let us neglect the residual $3 \mathrm{NF}$ terms of Eq. (3) and perform $\operatorname{CCSD}(\mathrm{T})$ calculations for the binding energy of ${ }^{4} \mathrm{He}$. The approximate inclusion of three-particle-threehole clusters improves the accuracy of our calculations. Our results are shown in Fig. 8 . The comparison with

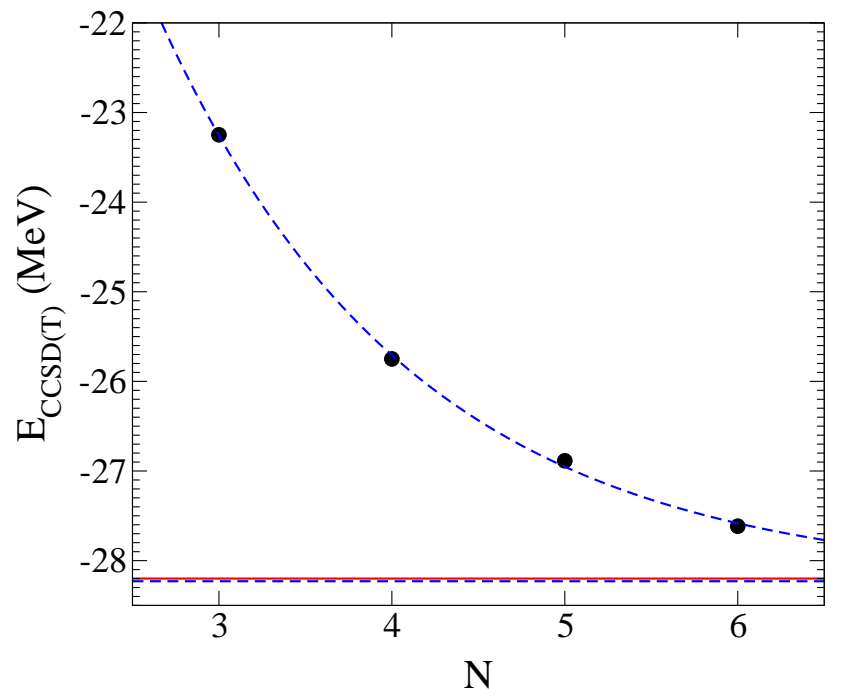

FIG. 9: (Color online) Data points: CCSD(T) results (taken at the $\hbar \omega$ minima) for the binding energy of ${ }^{4} \mathrm{He}$ with $3 \mathrm{NFs}_{\mathrm{s}}$ as a function of the number of oscillator shells. Dashed lines: Exponential fit to the data and asymptote of the fit. Full line: Exact result.

the CCSD full lines in Fig. 5 shows that the triples corrections add about $100-200 \mathrm{keV}$ of additional binding energy (at the minimum) for fixed number $N$ of oscillator shells, and somewhat weakens the $\hbar \omega$ dependence.

An exponential extrapolation of the (approximate) $\operatorname{CCSD}(\mathrm{T})$ minima to an infinite model space is shown in Fig. 9 and yields $E_{\infty}=-28.24 \mathrm{MeV}$. This is in excellent agreement with the exact Faddeev-Yakubovsky result $E=-28.20(5) \mathrm{MeV}$. In our largest model space at the minimum $\hbar \omega=17 \mathrm{MeV}$, the ground-state expectation values for the center-of-mass Hamiltonian is $\left\langle H_{\mathrm{cm}}\right\rangle \approx 20 \mathrm{keV}$ while the expectaion value for the angular momentum is zero for a closed-shell nucleus by construction. These results are very good, and it remains to be seen whether a more sophisticated treatment of triples excitations [63] would lead to further improvements. We expect that the expectation value of the center-of-mass Hamiltonian decreases with increasing size of the model space.

Finally, we also show the size of the CCSD(T) corrections in Fig. 7 as the horizontal dotted line. Clearly, these contributions are more important than the contributions from the residual $3 \mathrm{NF}$ terms, and this observation fully justifies the omission of the latter.

\section{SUMMARY}

We have developed coupled-cluster theory for threebody Hamiltonians in the two-particle-two-hole cluster approximation (CCSD). We derived the corresponding coupled-cluster equations directly in a factorized form and thereby avoided the explicit construction and analysis of a considerable number of diagrams that enter these 
equations. The resulting formulae were used for a very efficient numerical implementation.

We have performed ab-initio coupled-cluster calculations based on low-momentum NN and $3 \mathrm{~N}$ interactions for the binding energy of ${ }^{4} \mathrm{He}$ and compared to the exact Faddeev-Yakubovsky result. The 3NF contributions to the zero-, one-, and two-body terms of the normalordered Hamiltonian are dominant. The contributions from residual 3NFs are smaller than the corrections due to three-particle-three-hole cluster excitations and can therefore be safely neglected. Future work will include all $3 \mathrm{~N}$ partial waves and studies of the cutoff dependence and of the convergence properties using low-momentum interactions with smooth cutoffs. Our findings tremendously simplify the computational cost of coupled-cluster theory with $3 \mathrm{NFs}$. This opens the avenue to explore $3 \mathrm{NFs}$ in medium-mass nuclei and to investigate questions related to modern nuclear interactions.

\section{Acknowledgments}

We thank D. Bernholdt, S.K. Bogner, R.J. Furnstahl and R.J. Harrison for useful discussions. This research was supported in part by the Laboratory Directed Research and Development program of Oak Ridge National Laboratory (ORNL), by the U.S. Department of Energy under Contract Nos. DE-AC0500OR22725 with UT-Battelle, LLC (ORNL), and DEFC02-07ER41457 (University of Washington), and under Grant Nos. DE-FG02-96ER40963 (University of Tennessee), DE-FG02-97ER41014 (University of Washington), DE-FG02-01ER15228 (Michigan State University), and by the Natural Sciences and Engineering Research Council of Canada (NSERC). TRIUMF receives federal funding via a contribution agreement through the $\mathrm{Na}$ tional Research Council of Canada. Computational resources were provided by the National Center for Computational Sciences at Oak Ridge, the National Energy Research Scientific Computing Facility, and the John von Neumann Institute for Computing in Jülich, Germany.
[1] H. Kamada, A. Nogga, W. Glöckle, E. Hiyama, M. Kamimura, K. Varga, Y. Suzuki, M. Viviani, A. Kievsky, S. Rosati, J. Carlson, S.C. Pieper, R.B. Wiringa, P. Navrátil, B.R. Barrett, N. Barnea, W. Leidemann, and G. Orlandini, Phys. Rev. C 64, 044001 (2001), nucl-th/0104057.

[2] S.C. Pieper and R.B. Wiringa, Ann. Rev. Nucl. Part. Sci. 51, 53 (2001), nucl-th/0103005

[3] R.B. Wiringa and S.C. Pieper, Phys. Rev. Lett. 89, 182501 (2002), nucl-th/0207050

[4] A. Nogga, H. Kamada, W. Glöckle, and B.R. Barrett, Phys. Rev. C 65, 054003 (2002), nucl-th/0112026.

[5] P. Navrátil, J.P. Vary, and B.R. Barrett, Phys. Rev. C 62, 054311 (2000).

[6] P. Navrátil and W.E. Ormand, Phys. Rev. C 68, 034305 (2003), nucl-th/0305090

[7] A. Nogga, P. Navrátil, B.R. Barrett and J.P. Vary, Phys. Rev. C 73, 064002 (2006), nucl-th/0511082.

[8] G.P. Lepage, "How to Renormalize the Schrödinger Equation", Lectures given at 9th Jorge Andre Swieca Summer School: Particles and Fields, Sao Paulo, Brazil, February, 1997, nucl-th/9706029.

[9] F. Coester, Nucl. Phys. 7, 421 (1958).

[10] F. Coester and H. Kümmel, Nucl. Phys. 17, 477 (1960).

[11] J. Čížek, J. Chem. Phys. 45, 4256 (1966).

[12] J. Čížek, Adv. Chem. Phys. 14, 35 (1969).

[13] H. Kümmel, K.H. Lührmann, and J.G. Zabolitzky, Phys. Rep. 36, 1 (1978).

[14] R.J. Bartlett, J. Phys. Chem. 93, 1697 (1989).

[15] J. Paldus and X. Li, Adv. Chem. Phys. 110, 1 (1999).

[16] T.D. Crawford and H.F. Schaefer III, Rev. Comput. Chem. 14, 33 (2000).

[17] P. Piecuch, K. Kowalski, I.S.O. Pimienta, P.-D. Fan, M.
Lodriguito, M.J. McGuire, S.A. Kucharski, T. Kus, and M. Musial, Theor. Chem. Acc. 112, 349 (2004).

[18] J.H. Heisenberg and B. Mihaila, Phys. Rev. C 59, 1440 (1999), nucl-th/9802029.

[19] B. Mihaila and J.H. Heisenberg, Phys. Rev. C 61, 054309 (2000), nucl-th/9912023; Phys. Rev. Lett. 84, 1403 (2000), nucl-th/9910007.

[20] D.J. Dean and M. Hjorth-Jensen, Phys. Rev. C 69, 054320 (2004), nucl-th/0308088.

[21] K. Kowalski, D.J. Dean, M. Hjorth-Jensen, T. Papenbrock, and P. Piecuch, Phys. Rev. Lett. 92, 132501 (2004), nucl-th/0310082

[22] M. Włoch, D.J. Dean, J.R. Gour, M. Hjorth-Jensen, K. Kowalski, T. Papenbrock, and P. Piecuch, Phys. Rev. Lett. 94, 212501 (2005), nucl-th/0501067.

[23] J.R. Gour, P. Piecuch, M. Hjorth-Jensen, M. Włoch, and D.J. Dean, Phys. Rev. C 74, 024310 (2006), nucl-th/0507049.

[24] G. Hagen, D.J. Dean, M. Hjorth-Jensen, and T. Papenbrock, nucl-th/0610072

[25] F. Wegner, Ann. Physik, Leipzig 3, 77 (1994).

[26] S.D. Glazek and K.G. Wilson, Phys. Rev. D 49, 4214 (1994)

[27] S.R. White, cond-mat/0201346.

[28] S.A. Coon, M.D. Scadron, P.C. McNamee, B.R. Barrett, D.W.E. Blatt, and B.H.J. McKellar, Nucl. Phys. A 317, 242 (1979).

[29] S.A. Coon and M.T. Pena, Phys. Rev. C 48, 2559 (1993), nucl-th/9305017.

[30] J. Fujita and H. Miyazawa, Prog. Theo. Phys. 17, 360 (1957).

[31] M.R. Robilotta and H.T. Coelho, Nucl. Phys. A 460, 645 (1986). 
[32] J.A. Eden and M.F. Gari, Phys. Rev. C 53, 1510 (1996).

[33] B.S. Pudliner, V.R. Pandharipande, J. Carlson, S.C. Pieper, and R.B. Wiringa, Phys. Rev. C 56, 1720 (1997), nucl-th/9705009

[34] S.C. Pieper, V.R. Pandharipande, R.B. Wiringa and J. Carlson, Phys. Rev. C 64, 014001 (2001), nucl-th/0102004.

[35] P.F. Bedaque and U. van Kolck, Ann. Rev. Nucl. Part. Sci. 52, 339 (2002), nucl-th/0203055.

[36] E. Epelbaum, Prog. Part. Nucl. Phys. 57, 654 (2006), nucl-th/0509032.

[37] D.R. Entem and R. Machleidt, Phys. Rev. C 68, 041001(R) (2003), nucl-th/0304018

[38] E. Epelbaum, W. Glöckle, and U.G. Meißner, Nucl. Phys. A 747, 362 (2005), nucl-th/0405048.

[39] S. Weinberg, Phys. Lett. B 251, 288 (1990); Nucl. Phys. B 363, 3 (1991).

[40] U. van Kolck, Phys. Rev. C 49, 2932 (1994).

[41] J.L. Friar, D. Hüber and U. van Kolck, Phys. Rev. C 59, 53 (1999), nucl-th/9809065.

[42] E. Epelbaum, A. Nogga, W. Glöckle, H. Kamada, U.G. Meißner and H. Witala, Phys. Rev. C 66, 064001 (2002), nucl-th/0208023

[43] C. Ordonez, L. Ray and U. van Kolck, Phys. Rev. C 53, 2086 (1996), hep-ph/9511380

[44] E. Epelbaum, W. Glöckle and U.G. Meißner, Nucl. Phys. A 671, 295 (2000), nucl-th/9910064.

[45] P. Navrátil and E. Caurier, Phys. Rev. C 69, 014311 (2004), nucl-th/0311036.

[46] C. Forssen, P. Navrátil, W.E. Ormand, and E. Caurier, Phys. Rev. C 71, 044312 (2005), nucl-th/0412049.

[47] P. Navrátil, V.G. Gueorguiev, J.P. Vary, W.E. Ormand, and A. Nogga, nucl-th/0701038.
[48] S.K. Bogner, T.T.S. Kuo, A. Schwenk, D.R. Entem, and R. Machleidt, Phys. Lett. B 576, 265 (2003), nucl-th/0108041.

[49] S.K. Bogner, T.T.S. Kuo, and A. Schwenk, Phys. Rept. 386, 1 (2003), nucl-th/0305035.

[50] A. Nogga, S.K. Bogner, and A. Schwenk, Phys. Rev. C 70, 061002(R) (2004), nucl-th/0405016

[51] S.K. Bogner, A. Schwenk, R.J. Furnstahl, and A. Nogga, Nucl. Phys. A 763, 59 (2005), nucl-th/0504043

[52] A. Schwenk, J. Phys. G31, S1273 (2005), nucl-th/0412052.

[53] S.A. Kucharski and R.J. Bartlett, Adv. Quant. Chem. 18, 281 (1986).

[54] S.A. Kucharski and R.J. Bartlett, Theor. Chim. Acta 80, 387 (1991).

[55] S.A. Kucharski and R.J. Bartlett, J. Chem. Phys. 97, 4282 (1992).

[56] P. Piecuch, S.A. Kucharski, K. Kowalski, and M. Musiał, Comp. Phys. Comm. 149, 71 (2002).

[57] R.B. Wiringa, V.G.J. Stoks, and R. Schiavilla, Phys. Rev. C 51, 38 (1995).

[58] S.K. Bogner, R.J. Furnstahl, S. Ramanan, and A. Schwenk, Nucl. Phys. A 784, 79 (2007), nucl-th/0609003.

[59] P. Navrátil and W.E. Ormand, Phys. Rev. Lett. 88, 152502 (2002).

[60] A.P. Zuker, Phys. Rev. Lett. 90, 042502 (2003), nucl-th/0209073.

[61] J.W. Negele, Rev. Mod. Phys. 54, 913 (1982).

[62] M.J.O. Deegan and P.J. Knowles, Chem. Phys. Lett. 227, 321 (1994).

[63] P. Piecuch and M. Włoch, J. Chem. Phys. 123, 224105 (2005). 